\title{
28 The seventh cranial nerve
}

A The intracranial segment can be exposed through a posterior fossa craniectomy.

B The stylomastoid foramen can be visualized in the submentovertical view of the skull base.

C The junction of the facial canal with the lateral semicircular canal is usually marked by a mucosal vein.

D The anterior end of the digastric ridge is a useful landmark for the stylomastoid foramen.

E The main trunk is usually divided and grafted during superficial parotidectomy.

\section{Physical properties of sound}

A Frequency is subjectively perceived as pitch.

$B$ Intensity and loudness are related to sound energy.

C The reference intensity pressure in audiometry is 0.024 dyne/ $\mathrm{cm}^{2}$ at $100 \mathrm{~Hz}$.

D Overtones are multiples of the fundamental note.

E White noise is produced by many frequencies at different intensities.

\section{Sound transmission in the middle ear}

A The intact tympanic membrane protects the round window and directs sound energy to the ossicular chain and oval window.

$B$ The ossicular leverage action ratio of the malleus and incus is about 1.3:1.

C The mode of vibration of the stapes changes with high sound intensities.

D The physiological ratio of tympanic membrane to oval window surface area is about $21: 1$.

E The transformer ratio of the ossicular chain plus the tympanic membrane is about $18: 1$.

\section{Middle ear acoustic impedance}

A An increase in the stiffness of the vibrating parts produces frequency-selective deafness.

B The presence of fluid affects mainly the high frequencies.

C Ossicular chain discontinuity with an intact tympanic membrane results in a reduced middle ear compliance.

D Otosclerosis and adhesive otitis media increase the stiffness of the vibrating parts.

E Tympanosclerosis increases the middle ear compliance. 